\title{
Physical activity level and burden of malnutrition among farmers of selected agroecological zone of Bangladesh
}

\author{
Tasnima Mahjabin ${ }^{1}$, Ahmed Jubayer ${ }^{2 *}$, Kazi Abul Kalam ${ }^{1}$, Elora Parvin ${ }^{1}$, Md. Shamsuzzoha ${ }^{1}$, Md. Nazrul Islam \\ Khan $^{2}$, Md. Hafizul Islam ${ }^{2}$ \\ ${ }^{1}$ Bangladesh Institute of Research and Training on Applied Nutrition (BIRTAN), Dhaka, Bangladesh \\ ${ }^{2}$ Institute of Nutrition and Food Science, University of Dhaka, Dhaka-1000, Bangladesh
}

Email:

TM: mahjabin_79@yahoo.com

AJ: jubayerinfs@gmail.com

KAK: kaziabulkalam@gmail.com

EP: elorasau@gmail.com

MS: kbdzoha@gmail.com

NIK: nik_infs@du.ac.bd

HI: hafizinfs19@gmail.com

\begin{abstract}
*Corresponding author: Ahmed Jubayer, Institute of Nutrition and Food Science, University of Dhaka, Dhaka-1000, Bangladesh. Email: jubayerinfs@ @mail.com; Phone: +8801941232064; ORCID id: https://orcid.org/0000-0001-8369-8413
\end{abstract}

DOI: 10.31364/SCIRJ/v8.i12.2020.P1220831

http://dx.doi.org/10.31364/SCIRJ/v8.i12.2020.P1220831

\begin{abstract}
Agricultural productivity is largely dependent on nutritional status of farmers who are the central of agricultural development. This study aimed to assess prevalence and predictors of different forms of malnutrition and physical activity level (PAL) among farmers of selected agroecological zone of Bangladesh. The study was conducted in two subdistricts of $8^{\text {th }}$ agroecological zone of Bangladesh. 360 households were selected randomly and printed version of questionnaire was used to record necessary information. Nutritional status was assessed by calculating Body Mass Index (BMI) and physical activity level was measured based on self-reported information. Anthropometric information was collected for 357 farmers. Mean age, weight and height was $52 \pm 12$ years, $58.45 \pm 10 \mathrm{~kg}$ and $161.76 \pm 5.89 \mathrm{~cm}$ respectively. About one-third $(34.7 \%)$ of study participants were identified as overweight, $12.9 \%$ as underweight and $6.7 \%$ farmers were obese. Prevalence of overweight and obesity was higher among younger farmers (<49years). Odds of being overweight was 3.54times higher at Singair (AOR:3.54, p<0.0001) as compared to farmers of Sreepur upazila. Other covariates for overweight were young farmer (AOR: 2.31, $\mathrm{p}=0.008$ ) and illiterate farmers (AOR:0.49, $\mathrm{p}=0.027$ ). Mean PAL value and total energy expenditure was $2.2 \pm 0.05$ and $3641.77 \pm 56 \mathrm{kcal}$ respectively. Quite similarities were observed between farmers of two residential area although proportion of farmers with excessive physical activity was higher at Sreepur. Burden of overnutrition and physical activity level was correspondingly high among farmers with poor dietary diversity and low level of education. Appropriate intervention strategies fostering proper access to health information and health education for famers may be needed.
\end{abstract}

Keywords: Agroecological zone, malnutrition, physical activity, farmers, Bangladesh

\section{Introduction}

Bangladesh is one of the most populous countries in the world with high population density of 1063 person per square kilometer[1] which is now a lower middle-income country[2] with per capita Gross Domestic Product (GDP) \$1970 in financial year 2019-20[3]. Traditionally Bangladesh has a distinct agricultural setting where 60 percent population reside in rural areas and almost 88 percent of them are directly or indirectly engaged in agriculture[1]. The enormous contribution of agriculture sector on country's economy is

www.scirj.org

(C) 2020, Scientific Research Journal

http://dx.doi.org/10.31364/SCIRJ/v8.i12.2020.P1220831

This publication is licensed under Creative Commons Attribution CC BY. 
indisputable since it contributes almost 15percent of GDP[1]. Moreover, agriculture sector is important to achieve sustainable food and nutrition security of its large population.

Despite a small country, Bangladesh has different land types is different areas. Basically, there are five types of land level in Bangladesh: highland, medium high land, medium low land, low land and very low land[4]. An agroecological Zone (AEZ) is a zone or region with a unique combination of physiographic, soil, hydrological and agroclimatic characteristics[5]. For this reason, cropping system of a definite AEZ is almost same. Thirty Agroecological Zones (AEZs), 88 sub regions and 535 agroecological units have been identified in Bangladesh. The present study targeted famers of $8^{\text {th }}$ AEZ (Young Brahmpaputra and Jamuna floodplain).

The farmers and/or agriculture labors are the central of agricultural development. Regardless of enormous contribution in country's economy, a very little is known about dietary practice, nutritional status and physical activity level of farmers since these issues are not well studied. In fact, farmer's nutritional status and health hygiene are not very prioritized. But there is no way to ignore these issues since, they are related to their occupational potentiality as well as sustainability of production. It is noteworthy that the recent National Agriculture policy 2018 has given importance on improvement of food and nutrition security as well as diversification of foods along with socio economic improvements of farmers[6]. To our knowledge, our study is the first ever to explore different forms of malnutrition and physical activity level among farmers of agroecological zone of Bangladesh. Moreover, this study is aligned with National Nutrition Policy -2015.

Bangladesh Integrated Household Survey (BIHS) is the only nationally representative survey for rural Bangladesh that collect detail anthropometric information of rural population. Reported mean weight and height of elderly population in baseline survey of BIHS is $45.59 \mathrm{~kg}$ and $153.5 \mathrm{~cm}[7]$. BIHS also reported that 35.3 percent rural households are energy deficient. According to Nutrition, health and demographic survey of Bangladesh [8], 40.3percent old aged people are underweight in rural Bangladesh. Physical activity level data is very rare in Bangladesh because very few studies introduce theses challenging task in their studies. Nutrition, health and demographic survey -2011 is the unique survey in these regards that measured physical activity level and energy balance of Bangladeshi population. This national survey estimated mean physical activity level (PAL) 2.0 for farmers/fisherman. Both of these national surveys are related to our study but they represent the whole country whereas our study is a small-scale study that covers only a subregion of $8^{\text {th }}$ AEZ. Moreover, we can get an idea about nutritional status and physical activity level of farmers of Bangladesh but still there is a research gap exists. Our study covered all of these issues since primary objective of the present study was to examine the burden of malnutrition and physical activity pattern among farmers of selected sub regions of $8^{\text {th }}$ AEZ. Furthermore, through suitable statistical statistics, their socio-economic determinants were also identified.

\section{Methodology}

Study design and location: The study adopted cross sectional study design to record necessary information from targeted population. Among 30 Agro- ecological zone (AEZ) of Bangladesh, 8 th AEZ (Young Brahmaputra-Jamuna floodplain) was chosen for this study. The study conducted in two sub-districts of Manikganj and Gazipur district.

Sample size: Prevalence of underweight among old age people in Dhaka division is $35 \%$ as estimated by Nutrition, health and demographic survey of Bangladesh-2011.The sample size for this study was determined by using the following formula: $n=\left(z^{2}\right.$ $\left.{ }^{*} \mathrm{p} * \mathrm{q}\right) / \mathrm{d}^{2}$ where $\mathrm{n}=$ minimum sample size for a specified population, $\mathrm{p}=$ prevalence of malnutrition among old aged people of Dhaka division which is $35 \%, \mathrm{~d}=$ Margin of error or precision level $=0.05$ and $\mathrm{Z}=$ the standard normal deviation which is 1.96 at $95 \%$ confidence interval. Minimum sample size was calculated 350 following this equation. Finally, 360 farmers were sampled in this study.

Sampling method: Two upazilas from Manikganj and Gazipur district (one upazila from each district) were chosen purposively for inclusion in the study. Following this, two unions from Singair upazila and one union from Sreepur upazila were randomly selected. Then three villages were randomly selected from two union of Singair and four villages from one union of Sreepur upazila, to yield a total of seven villages from which survey households were selected. Households were selected randomly and total required number of households were distributed in 2 upazilas (50percent from each upazila). Number of households from each village are given in table 1.

Table 1: Sample distribution across study locations

\begin{tabular}{|c|c|c|c|c|c|}
\hline \multirow[t]{2}{*}{ AEZ } & \multicolumn{4}{|c|}{ Area Covers } & \multirow{2}{*}{$\begin{array}{c}\text { Number of } \\
\text { households sample }\end{array}$} \\
\hline & District & Upazila & Union & Village & \\
\hline 8. Young & & & Joymontap & Durgapur & 91 \\
\hline Brahmaputra- & Manikganj & Singair & & & \\
\hline Jamuna & & & Dholla & Ulail & 48 \\
\hline floodplain & & & & Khaser char & 41 \\
\hline
\end{tabular}

www.scirj.org

(C) 2020, Scientific Research Journal

http://dx.doi.org/10.31364/SCIRJ/v8.i12.2020.P1220831

This publication is licensed under Creative Commons Attribution CC BY. 


\begin{tabular}{|c|c|c|c|c|}
\hline Gazipur & Sreepur & Bormi & Borpur & 74 \\
\hline & & & Borma & 46 \\
\hline & & & Kaet para & 44 \\
\hline & & & Mudibari & 16 \\
\hline
\end{tabular}

Data collection: Printed version of questionnaire was used to record necessary information from study subjects. Questionnaire was pretested prior to final field survey. A well-qualified survey team was appointed for data collection. Portable weight and height scale were used to record anthropometric information.

Assessment of nutritional status: Asian specific Body Mass Index (BMI) cut-offs were used to define underweight $\left(<18.5 \mathrm{~kg} / \mathrm{m}^{2}\right)$, overweight $\left(23.0\right.$ to $\left.<27.5 \mathrm{~kg} / \mathrm{m}^{2}\right)$ and obese $\left(\geq 27.5 \mathrm{~kg} / \mathrm{m}^{2}\right.$ [9]

Determination of physical activity level and total energy expenditure: The enumerators recorded different physical activity and duration of these activities that the subjects perform previous day. Physical activity level (PAL) of an individual was determined by following equation:

$$
\mathrm{PAL}=\frac{\Sigma \text { duration of activity } \times \text { average } P A R}{1440}
$$

Physical activity level was classified into 3 categories as proposed by FAO/WHO/UNU expert consultation( [10] ) : Sedentary or light physical activity (PAL 1.4 TO 1.69), active or moderately active (PAL 1.7 to1.99), vigorous or vigorously active lifestyle (2 to 2.4). Basal Metabolic Rate (BMR) was calculated prior to estimate Total Energy Expenditure (TEE) using Harris Benedict equation.

Male: $\mathrm{BMR}=66.1+(13.5 \times$ weight in $\mathrm{kg}+(5 \times$ Height in $\mathrm{cm})-(6.75 \times$ age $)$

Female: $\mathrm{BMR}=665+(9.6 \times$ weight in $\mathrm{kg})+(1.7 \times$ height in $\mathrm{cm})-(4.7 \times$ age $)$

Then TEE was calculated using the formula: TEE $=\mathrm{BMR} \times \mathrm{PAL}$

Statistical analysis: All statistical analyses were performed using SPSS (version 25). Prevalence of different forms of malnutrition was calculated with 95\% confidence interval. Chi square test was used estimating the association between selected factors and nutritional status. We also fitted multinominal logistics regression model to estimate the effect of different factors on nutritional status. All possible covariates were included into regression model. Nutritional status of farmers was considered as response variable categorized into 3 group: underweight, overweight and normal. Nutritional status "normal" was considered as reference. All statistical significance was defined as $\mathrm{p}$ value less than 0.05 in two tailed tests.

\section{Results}

Characteristics of study subjects: Household and individual demographic and socio-economic characteristics are listed in table 2 . In total 360 farmers from were included in the current study. Almost two-fifth (39.4\%) were aged less than 49 years and $30.3 \%$ participants were old age. Proportion of old age people were significantly higher at Sreepur upazila. Literacy level was observed very low; $57.3 \%$ farmers had no education and only $19.7 \%$ had primary education. A distinct regional variation was also noticed with higher level of illiteracy at Singair upazila. Average household size was 4.49 as though about $50 \%$ farmers household comprising less than 5 persons. The sampled farmers were disaggregated into four operated farm size groups. More than half of them were medium farmer and $17.2 \%$ were small farmer. At the other extreme, only $6.7 \%$ were large farmers. Percentage of small medium farmers were higher at Sreepur upazila. On the other hand, proportion of large farmers were higher at Singair. In case of sanitatin facility, more than two-third of sampled household had improved sanitation facility; this percentage is high at Singair. Most of the household was food secure measured by Food consumption score as $95.3 \%$ had acceptable score. A remarkable proportion of farmers had low diversified $\operatorname{diet}(59.7 \%)$.

www.scirj.org

(C) 2020, Scientific Research Journal

http://dx.doi.org/10.31364/SCIRJ/v8.i12.2020.P1220831

This publication is licensed under Creative Commons Attribution CC BY. 
Table 2: General characteristics of study subjects

\begin{tabular}{|c|c|c|c|c|}
\hline & $\begin{array}{l}\text { Total } \\
\text { n(\%) }\end{array}$ & $\begin{array}{c}\text { Singair } \\
\mathbf{n}(\%)\end{array}$ & $\begin{array}{l}\text { Sreepur } \\
\text { n(\%) }\end{array}$ & p value \\
\hline $\begin{array}{l}\text { Age in year } \\
<49 \\
49 \text { to } 60 \\
>60\end{array}$ & $\begin{array}{l}142(39.4) \\
109(30.3) \\
109(30.3)\end{array}$ & $\begin{array}{l}79(43.9) \\
52(28.9) \\
49(27.2)\end{array}$ & $\begin{array}{c}63(35) \\
57(31.7) \\
60(33.3)\end{array}$ & 0.08 \\
\hline $\begin{array}{l}\text { Level of education } \\
\text { No education } \\
\text { Primary or below } \\
\text { Secondary or higher } \\
\text { Missing }\end{array}$ & $\begin{array}{c}204(57.3) \\
71(19.7) \\
81(22.5) \\
4(1.1)\end{array}$ & $\begin{array}{c}108(60) \\
29(16.11) \\
40(22.23) \\
3(1.67)\end{array}$ & $\begin{array}{c}96(53.33) \\
42(23.33) \\
41(22.78) \\
1(0.56)\end{array}$ & 0.38 \\
\hline $\begin{array}{l}\text { Household size } \\
\leq 4 \\
5 \\
6+\end{array}$ & $\begin{array}{l}184(51.1) \\
90(25) \\
86(23.9)\end{array}$ & $\begin{array}{l}96(53.3) \\
46(25.6) \\
38(21.1)\end{array}$ & $\begin{array}{l}88(48.9) \\
44(24.4) \\
48(26.7)\end{array}$ & 0.24 \\
\hline $\begin{array}{l}\text { Income quintile } \\
\text { Lowest }(\leq 18100 \mathrm{tk}) \\
\text { Second }(18101-23000 \mathrm{tk}) \\
\text { Third }(23001-29000 \mathrm{tk}) \\
\text { Fourth }(29001-36630 \mathrm{tk}) \\
\text { Highest }(\geq 36631 \mathrm{tk})\end{array}$ & $\begin{array}{c}72(20) \\
72(20) \\
71(19.7) \\
72(20) \\
71(19.7)\end{array}$ & $\begin{array}{c}35(19.6) \\
34(19) \\
25(14) \\
31(17.3) \\
54(30.2)\end{array}$ & $\begin{array}{c}37(20.7) \\
38(21.2) \\
46(25.7) \\
41(22.9) \\
17(9.5)\end{array}$ & 0.007 \\
\hline $\begin{array}{l}\text { Farm size group } \\
\text { Marginal }(<0.5 \text { acres }) \\
\text { Small }(0.5-1.49 \text { acres }) \\
\text { Medium }(1.5-2.49 \text { acres }) \\
\text { Large }(\geq 2.5 \text { acres })\end{array}$ & $\begin{array}{c}62(17.2) \\
203(56.4) \\
71(19.7) \\
24(6.7)\end{array}$ & $\begin{array}{c}31(17.2) \\
83(46.1) \\
48(26.7) \\
18(10)\end{array}$ & $\begin{array}{c}31(17.2) \\
120(66.7) \\
23(12.8) \\
6(3.3)\end{array}$ & $<0.0001$ \\
\hline $\begin{array}{l}\text { Sanitation facility } \\
\text { Sanitary latrine } \\
\text { Other }\end{array}$ & $\begin{array}{l}248(68.9) \\
112(31.1)\end{array}$ & $\begin{array}{c}127(70.6) \\
53(29.4)\end{array}$ & $\begin{array}{c}121(67.2) \\
59(32.8)\end{array}$ & 0.56 \\
\hline $\begin{array}{l}\text { FCS category } \\
\text { Poor and borderline } \\
\text { Acceptable }\end{array}$ & $\begin{array}{c}17(4.7) \\
343(95.3)\end{array}$ & $\begin{array}{c}12(6.7) \\
168(93.3)\end{array}$ & $\begin{array}{c}5(2.8) \\
175(97.2)\end{array}$ & 0.13 \\
\hline $\begin{array}{l}\text { Dietary Diversity score } \\
\leq 5 \\
\geq 6\end{array}$ & $\begin{array}{l}215(59.7) \\
145(40.3)\end{array}$ & $\begin{array}{c}100(55.6) \\
80(44.4)\end{array}$ & $\begin{array}{c}115(63.9) \\
65(36.1) \\
\end{array}$ & 0.13 \\
\hline
\end{tabular}

Burden of malnutrition among farmers: Proportion of farmers classified as different forms of malnutrition is presented in table 3 . About one third (34.7\%) of them were identified as overweight, $12.9 \%$ as underweight and only $6.7 \%$ farmers were obese. Roughly, one in ten youngers $(\leq 49$ years) was underweight which is significantly higher than that of older farmers $(17.8 \%)$. Besides, onequarter on older farmer were overweight while younger persons were more likely to be overweight (45.8\%). At Sreepur, underweight prevalence was significantly higher overweight prevalence was lower. Overweight prevalence was more than double at Singair upazila. Literate farmers were more likely to be overweight and obese. In addition, proportion of farmers with low BMI classified as underweight was higher among marginal farmers and large farmers were more likely to be obese. As expected, both overweight and obesity was highly prevalent among richest persons. Moreover, Farmers having improved sanitary facility had low occurrence of underweight. 
Table 3: Cross classification type of malnutrition

\begin{tabular}{|c|c|c|c|}
\hline Variable & $\begin{array}{c}\text { Underweight }(\mathrm{BMI}<18.5 \\
\left.\mathrm{kg} / \mathrm{m}^{2}\right)\end{array}$ & $\begin{array}{l}\text { Overweight (BMI: } 23 \text { to } \\
\left.<27.5 \mathrm{~kg} / \mathrm{m}^{2}\right)\end{array}$ & Obese $\left(\mathrm{BMI} \geq 27.5 \mathrm{~kg} / \mathrm{m}^{2}\right)$ \\
\hline & $\%(95 \% \mathrm{CI})$ & $\%(95 \% \mathrm{CI})$ & $\%(95 \% \mathrm{CI})$ \\
\hline Total & $12.9(9.43,16.38)$ & $34.7(29.77,39.64)$ & $6.7(4.11,9.3)$ \\
\hline $\begin{array}{l}\text { Age in year } \\
<49 \\
49 \text { to } 60 \\
>60\end{array}$ & $\begin{array}{c}9.2(4.45,13.96) \\
13(6.66,19.35) \\
17.8(10.56,25.05)\end{array}$ & $\begin{array}{c}45.8(37.61,54) \\
29.6(21,38.21) \\
25.2(16.98,33.43)\end{array}$ & $\begin{array}{c}8.5(3.92,13.09) \\
6.5(1.86,11.15) \\
4.7(0.69,8.72)\end{array}$ \\
\hline $\mathrm{p}$-value $\left(\chi^{2}\right)$ & 0.046 & 0.001 & 0.23 \\
\hline $\begin{array}{l}\text { Study location } \\
\text { Singair } \\
\text { Sreepur }\end{array}$ & $\begin{array}{c}7.2(3.43,10.98) \\
18.2(12.57,23.84)\end{array}$ & $\begin{array}{l}46.7(39.42,53.99) \\
22.6(16.49,28.72)\end{array}$ & $\begin{array}{l}10(5.62,14.39) \\
3.4(0.76,6.05)\end{array}$ \\
\hline $\mathrm{p}$-value $\left(\chi^{2}\right)$ & 0.001 & $<0.0001$ & 0.018 \\
\hline $\begin{array}{l}\text { Level of education } \\
\text { No education } \\
\text { Primary or below } \\
\text { Secondary or higher }\end{array}$ & $\begin{array}{c}14.9(9.9,19.82) \\
12.9(5.05,20.76) \\
8.6(2.5,14.71)\end{array}$ & $\begin{array}{c}30.7(24.34,37.07) \\
37.1(25.79,48.42) \\
42(31.26,52.75)\end{array}$ & $\begin{array}{c}4(1.3,6.71) \\
4.3(-0.46,9.06) \\
14.8(7.07,22.54)\end{array}$ \\
\hline $\mathrm{p}$-value $\left(\chi^{2}\right)$ & 0.16 & 0.062 & 0.002 \\
\hline $\begin{array}{l}\text { Household size } \\
\leq 4 \\
5 \\
6+\end{array}$ & $\begin{array}{c}13.7(8.71,18.7) \\
8.9(3.02,14.79) \\
15.3(7.87,22.74)\end{array}$ & $\begin{array}{l}34.6(27.69,41.52) \\
36.7(26.75,46.66) \\
32.9(22.92,42.89)\end{array}$ & $\begin{array}{c}4.4(1.43,7.38) \\
10(3.81,16.2) \\
8.2(2.37,14.04)\end{array}$ \\
\hline $\mathrm{p}$-value $(\chi 2)$ & 0.92 & 0.86 & 0.15 \\
\hline $\begin{array}{l}\text { Farm size group } \\
\text { Marginal }(<0.5 \text { acres }) \\
\text { Small }(0.5-1.49 \text { acres }) \\
\text { Medium }(1.5-2.49 \text { acres }) \\
\text { Large }(\geq 2.5 \text { acres })\end{array}$ & $\begin{array}{c}18(8.36,27.65) \\
11.9(7.43,16.38) \\
9.9(2.96,16.85) \\
16.7(1.78,31.63)\end{array}$ & $\begin{array}{c}32.8(21.02,44.59) \\
33.3(26.79,39.82) \\
30(19.35,40.66) \\
29.2(11.01,47.04)\end{array}$ & $\begin{array}{c}4.9(-0.52,10.32) \\
5.5(2.35,8.66) \\
7(1.07,12.94) \\
20.8(4.57,37.04)\end{array}$ \\
\hline $\mathrm{p}$-value $\left(\chi^{2}\right)$ & 0.48 & 0.58 & 0.029 \\
\hline $\begin{array}{l}\text { Sanitation facility } \\
\text { Sanitary latrine } \\
\text { Other }\end{array}$ & $\begin{array}{l}12.2(8.02,16.19) \\
14.3(7.82,20.79)\end{array}$ & $\begin{array}{c}36.7(30.67,42.74) \\
34(25.23,42.78)\end{array}$ & $\begin{array}{c}7.8(4.45,11.16) \\
4.5(0.68,8.33)\end{array}$ \\
\hline $\mathrm{p}-$ value $(\chi 2)$ & 0.61 & 0.28 & 0.36 \\
\hline $\begin{array}{l}\text { Income quintile } \\
\text { Lowest }(\leq 18100 \mathrm{tk}) \\
\text { Second }(18101-23000 \mathrm{tk}) \\
\text { Third }(23001-29000 \mathrm{tk}) \\
\text { Fourth }(29001-36630 \mathrm{tk}) \\
\text { Highest }(\geq 36631 \mathrm{tk})\end{array}$ & $\begin{array}{c}11.3(3.94,18.67) \\
17.1(8.28,25.93) \\
14.1(6.01,22.2) \\
15.3(6.99,23.62) \\
7(1.07,12.94)\end{array}$ & $\begin{array}{c}33.8(22.8,42.81) \\
28.6(18.02,39.19) \\
40.8(29.37,52.24) \\
34.7(23.71,45.7) \\
36.6(25.4,47.81)\end{array}$ & $\begin{array}{c}1.4(-1.34,4.14) \\
5.7(0.27,11.14) \\
7(1.07,12.94) \\
2.8(-1.02,6.62) \\
16.9(8.19,25.62)\end{array}$ \\
\hline $\mathrm{p}-$ value $\left(\chi^{2}\right)$ & 0.41 & 0.51 & 0.003 \\
\hline $\begin{array}{l}\text { FCS category } \\
\text { Poor and borderline } \\
\text { Acceptable }\end{array}$ & $\begin{array}{c}23.5(3.35,43.66) \\
12.4(8.9,15.91)\end{array}$ & $\begin{array}{l}29.4(7.75,51.06) \\
35(29.96,40.05)\end{array}$ & $\begin{array}{c}0.00 \\
7.1(4.39,9.82)\end{array}$ \\
\hline $\mathrm{p}$-value $(\chi 2)$ & 0.25 & 0.79 & 0.61 \\
\hline $\begin{array}{l}\text { Dietary Diversity score } \\
\leq 5 \\
\geq 6\end{array}$ & $\begin{array}{c}15.5(10.64,20.37) \\
9(4.33,13.68)\end{array}$ & $\begin{array}{l}33.8(27.45,40.16) \\
36.1(28.26,43.95)\end{array}$ & $\begin{array}{c}4.2(1.51,6.9) \\
10.4(5.42,15.39)\end{array}$ \\
\hline $\mathrm{p}$-value $\left(\chi^{2}\right)$ & 0.079 & 0.65 & 0.03 \\
\hline
\end{tabular}


Determinants of malnutrition: Multinominal logistic regression model was fitted to examine contribution of each explanatory variable on malnutrition. Table 4 presents the estimates of the assessment of logistic regression. Both categorical (fixed factors) and continuous variables (covariates) were included in regression model simultaneously to observe the effect of each explanatory factors on response variable. Odds of overweight was 3.54 times higher at Singair upazila (AOR: 3.54(95\%CI: 2.097,5.97; p<0.0001). An important thing to be noted that odds of being overweight was 2.32 times higher among younger farmers (AOR: 2.31(95\% CI: $1.242,4.297 ; \mathrm{p}=0.008)$. On the other hand, Odds of overweight was $51 \%$ lower among illiterate farmers $(\mathrm{AOR}=0.49,95 \% \mathrm{CI}: 0.26,0.923 ; \mathrm{p}<0.027)$.

Table 4: Multinominal logistic regression showing factors associated with malnutrition

\begin{tabular}{|c|c|c|c|c|}
\hline variable & $\begin{array}{c}\text { Underweight } \\
\text { AOR }(95 \% \mathrm{CI})\end{array}$ & p value & $\begin{array}{c}\text { Overweight } \\
\text { AOR }(95 \% \mathrm{CI})\end{array}$ & p value \\
\hline $\begin{array}{l}\text { Age in year } \\
<49 \\
49 \text { to } 60 \\
>60 \text { (reference) }\end{array}$ & $\begin{array}{l}0.722(0.304,1.716) \\
0.758(0.339,1.696)\end{array}$ & $\begin{array}{c}0.46 \\
0.5\end{array}$ & $\begin{array}{l}2.31(1.242,4.297) \\
1.32(0.694,2.511)\end{array}$ & $\begin{array}{c}0.008 \\
0.39\end{array}$ \\
\hline $\begin{array}{l}\text { Study area } \\
\text { Singair } \\
\text { Sreepur(reference) }\end{array}$ & $0.55(0.258,1.171)$ & 0.12 & $3.54(2.097,5.97)$ & $<0.0001$ \\
\hline $\begin{array}{l}\text { Level of education } \\
\text { No education } \\
\text { Primary or below } \\
\text { Secondary or higher(reference) }\end{array}$ & $\begin{array}{l}1.081(0.385,3.041) \\
1.006(0.313,3.229)\end{array}$ & $\begin{array}{l}0.88 \\
0.99\end{array}$ & $\begin{array}{c}0.49(0.26,0.923) \\
0.64(0.305,1.343)\end{array}$ & $\begin{array}{c}0.027 \\
0.21\end{array}$ \\
\hline $\begin{array}{l}\text { Dietary diversity score } \\
\leq 5 \\
\geq 6 \text { (reference })\end{array}$ & $1.482(0.648,3.389)$ & 0.35 & $1.153(0.654,2.032)$ & 0.62 \\
\hline $\begin{array}{l}\text { Sanitation facility } \\
\text { Sanitary latrine } \\
\text { Other(reference) }\end{array}$ & $1.316(0.606,2.861)$ & 0.48 & $1.157(0.65,2.06)$ & 0.62 \\
\hline Household size & $1.09(0.908,1.308)$ & 0.35 & $1.099(0.948,1.275)$ & 0.21 \\
\hline Area of cultivable land & $1.07(0.703,1.631)$ & 0.75 & $1.013(0.747,1.372)$ & 0.93 \\
\hline Food consumption score & $0.985(0.962,1.009)$ & 0.23 & $1.013(0.996,1.03)$ & 0.13 \\
\hline Household monthly income & $1.0(1.0,1.0)$ & 0.24 & $1.0(1.0,1.0)$ & 0.7 \\
\hline
\end{tabular}

Self-reported physical activity level of farmers: Table 5presents the distribution of farmers by mean PAL value and energy expenditure. Mean PAL value was estimated 2.2 (0.05) with minimum value of 0.24 and maximum value was 4.25. Mean energy expenditure of studied subjects was 3641.77 (893.56) kcal. Mean PAL value and energy expenditure level varied between two residential areas. The highest average PAL value was observed among farmer of Sreepur whereas average energy expenditure was higher at Singair. Quite similarities were found in term of average PAL value among small and medium farmers which was lower than that of marginal and large farmers. Besides, average energy expenditure was highest among large farmers. As expected, younger farmers obtained higher PAL value and their energy expenditure was also higher.

Table 5: Distribution of farmers by mean PAL value and energy expenditure

\begin{tabular}{lcccccc}
\hline & & PAL value & \multicolumn{2}{c}{ Energy Expenditure(kcal) } \\
\hline & Mean (SD) & Minimum & Maximum & Mean (SD) & Minimum & Maximum \\
\hline Upazila & & & & & & \\
Singair & $2.16(0.48)$ & 1.12 & 3.42 & $3673.49(857.03)$ & 1800.23 & 5919.34 \\
Sreepur & $2.24(0.51)$ & 0.24 & 4.25 & $3609.68(930.42)$ & 402.95 & 7675.63
\end{tabular}

www.scirj.org

(C) 2020, Scientific Research Journal

http://dx.doi.org/10.31364/SCIRJ/v8.i12.2020.P1220831

This publication is licensed under Creative Commons Attribution CC BY. 


\begin{tabular}{lcccccc} 
Farm size group & & & & & & \\
Marginal & $2.19(0.53)$ & 1.24 & 3.42 & $3557.1(938.69)$ & 1920.89 & 6079.29 \\
Small & $2.2(0.51)$ & 0.24 & 4.25 & $3624.43(915.38)$ & 402.95 & 7675.63 \\
Medium & $2.21(0.44)$ & 1.3 & 3.39 & $3705.3(802.67)$ & 2146.47 & 540.4 \\
Large & $2.19(0.45)$ & 1.23 & 2.92 & $3815.33(861.35)$ & 2238.54 & 5596.89 \\
& & & & & & \\
Age in year & & & & & & \\
$\leq 46$ & $2.27(0.54)$ & 1.14 & 4.25 & $3840.08(989.49)$ & 1586.13 & 7675.63 \\
47 to 60 & $2.15(0.45)$ & 1.16 & 3.42 & $3581.72(790.44)$ & 1832.38 & 5700.04 \\
$\geq 61$ & $2.19(0.48)$ & 0.24 & 3.5 & $3485.58(855.06)$ & 402.25 & 5885.15 \\
& & & & & & \\
Total & $\mathbf{2 . 2 ( 0 . 5 )}$ & $\mathbf{0 . 2 4}$ & $\mathbf{4 . 2 5}$ & $\mathbf{3 6 4 1 . 7 7 ( 8 9 3 . 5 6 )}$ & $\mathbf{4 0 2 . 9 5}$ & $\mathbf{7 6 7 5 . 6 3}$ \\
\hline
\end{tabular}

Table 6 depicts that famers were physically very much active since two third of them falls into vigorous and extremely high-level activity. Sustainable PAL value (1.4 to 2.4) for a long period of time was observed in remaining one third (approximately). Farmer from Sreepur upazila were physically more active and nearly 40 percent were observed extremely high-level activity. Percent of famers who falls into vigorous and extremely high-level activity was higher among medium sized farmers although quite similarities was found across farmers of different farm size. Proportion of younger farmers achieving extremely high level of physical activity was higher than older famers.

Table 6: Percent distribution of farmers according to PAL value by residence and farm size group

\begin{tabular}{|c|c|c|c|c|c|}
\hline & Low PAL $(<1.4)$ & $\begin{array}{l}\text { Sedentary or light } \\
\text { activity (1.4-1.69) }\end{array}$ & $\begin{array}{c}\text { Active or } \\
\text { moderate activity } \\
(1.7-1.99)\end{array}$ & $\begin{array}{l}\text { Vigorous } \\
\text { activity }(2-2.4)\end{array}$ & $\begin{array}{l}\text { High PAL } \\
(>2.4)\end{array}$ \\
\hline \multicolumn{6}{|l|}{ Upazila } \\
\hline Singair & 7.9 & 11.2 & 13.5 & 33.1 & 34.3 \\
\hline Sreepur & 6.7 & 7.3 & 13.4 & 33 & 39.7 \\
\hline \multicolumn{6}{|c|}{ Farm size group } \\
\hline Marginal & 6.5 & 12.9 & 17.7 & 22.6 & 40.3 \\
\hline Small & 7.9 & 8.4 & 12.4 & 33.7 & 37.6 \\
\hline Medium & 5.8 & 10.1 & 11.6 & 39.1 & 33.3 \\
\hline Large & 8.3 & 4.2 & 16.7 & 37.5 & 33.3 \\
\hline \multicolumn{6}{|c|}{ Age in year } \\
\hline$\leq 46$ & 5.7 & 9.8 & 12.3 & 29.5 & 42.6 \\
\hline 47 to 60 & 8.1 & 9.8 & 14.6 & 31.7 & 35.8 \\
\hline$\geq 61$ & 7.2 & 8.1 & 13.5 & 38.7 & 32.4 \\
\hline Total & 7.3 & 9.2 & 13.4 & 33.3 & 37 \\
\hline
\end{tabular}

\section{Discussion}

The main focus of the study was to estimate prevalence and predictors of malnutrition as well as physical activity level of farmers in two sub regions of $8^{\text {th }}$ agroecological zone of Bangladesh. Prevalence of underweight, overweight and obesity was $12.9 \%, 34.7 \%$ and $6.7 \%$ respectively. Odds of overweight was higher among younger and literate farmers as well. On the other hand, Farmers of Sreepur were more likely to be underweight. Physical activity level and energy expenditure of study subjects were quite high since almost one third of them were vigorously active.

Average size of farmers household was 4.49 which is slightly higher than the national estimates[11, 12]. Illiteracy rate was very much higher among our study subjects in compared to Household Income and Expenditure Survey (HIES)-2016 and Bangladesh Integrated Household Survey (BIHS) -2011[7, 13]. Farm size was calculated following cut off set by BIHS and HIES. BIHS reported that more

www.scirj.org

(C) 2020, Scientific Research Journal

http://dx.doi.org/10.31364/SCIRJ/v8.i12.2020.P1220831

This publication is licensed under Creative Commons Attribution CC BY. 
than one third of all farmers of rural Bangladesh were marginal farmers which is almost double than our estimation (17.2 percent). Besides, proportion of farmers classified as small, medium and large was almost same as recorded in BIHS -2011. Nationally, 86\% households had improved sanitation facility[14] which is higher than our estimation for farmers household.

In contrast to other studies[8, 15], overweight prevalence was little bit higher and underweight prevalence was lower although we couldn't compare our findings directly since we included only farmers from a subregions of $8^{\text {th }}$ AEZ and from literature we found no study that exactly match our study design. Additionally, our study identified appropriate predictors of malnutrition. Through logistic regression analysis, we found that place of residence, age and education level is significantly associated with malnutrition. Similar factors were identified significant association with nutritional status of household member of different age groups[16-18].

Physical activity level (PAL) is the second largest component of total energy expenditure which is important to maintain overall health and fitness and achieve energy balance. Total energy expenditure on the other hand reflects the average amount of energy spent in a typical day. Physical activity level and energy expenditure of farmers were very high which is an indication that their energy requirement is also high. These findings are in line with Nutrition, health and demographic survey of Bangladesh. [8].

A distinct regional variation was observed among farmers of two upazila (Singair and Sreepur) in overall dietary diversity, nutritional status and physical activity level. Low dietary diversity with high level of underweight prevalence was noticed among farmers of Sreepur upazila. On the other hand, diets were more diversified among farmers of Singair upazila and overweight prevalence was more than double in compare to Sreepur. Average energy expenditure and PAL value was almost equal in two strata although proportion of farmers with excessive physical activity was higher at Sreepur.

Geographical location and environmental condition may be responsible for this variation. The survey was conducted just at the onset of winter season. Recurrent flooding during rainy season in key agricultural production areas of Singair upazila led to widespread damage to crops and in rainy season people had to engaged in non-agricultural activities. On the other hand, Sreepur upazila was also affected by flood but not at the same as Singair upazila. Moreover, during the survey period, farmers were engaged mainly nonagricultural activities like grooming and tending domestic animals, cutting straw, small scale business etc.

\section{Strength and limitation}

A main strong point of this study was robust sample size that ensure proper representative of sample. Involvement of trained well experienced data collection team and use of validated questionnaire further increase the quality of data. Despite these, our study is not beyond of all kinds of limitation. Due to missing information regarding detailed dietary practice, we couldn't calculate energy balance. Furthermore, a single day measurement is not enough to predict usual physical activity pattern.

\section{Conclusion}

In addition to poor dietary practice, physical activity level and energy expenditure of farmers were extremely high. Most of the farmers were illiterate with no nutritional knowledge. Despite these, overweight and obesity prevalence was little bit higher. Appropriate intervention strategy may be needed to foster the farmers about healthier diet.

\section{Reference}

[1] BBS. BANGLADESH STRATEGIC PLAN ON AGRICULTURAL AND RURAL STATISTICS (2016-2030). 2017.

[2] World Bank. Bangladesh Overview, https://www.worldbank.org/en/country/bangladesh/overview (2015).

[3] BBS. Gross domestic product of Bangladesh at current price 2016-17 to 2019-20(p).

[4] Fao. LAND RESOURCES DATA BASE VOLUME II SOIL, LANDFORM AND HYDROLOGICAL DATA BASE /UNI TED NATIONS DEVELOPMENT PROGRAMME F A o FOOD AND AGRICULTURE ORGANIZATION $v J$ OF THE UNITED NATIONS. 1988.

[5] Agro-Ecological Zones -AEZs Maps, https://www.bamis.gov.bd/en/page/aezs-maps/.

[6] Ministry of Agriculture. National Agriculture policy 2018. 2019.

[7] Ahmed AU, Ahmad K, Chou V, et al. The Status of Food Security in the Feed the Future Zone and Other Regions of Bangladesh: Results from the 2011-2012 Bangladesh Integrated Household Survey. 2013.

[8] Akhtaruzzaman, Md Nazrul Islam Khan, Akter F. Nutrition, health and demographic survey of Bangladesh. 2013.

www.scirj.org

(C) 2020, Scientific Research Journal

http://dx.doi.org/10.31364/SCIRJ/v8.i12.2020.P1220831

This publication is licensed under Creative Commons Attribution CC BY. 
[9] WHO expert consultation. BMI for asian people. The lancet 2004; 363: 157-163.

[10] joint FAO/WHO/UNU expert consultation. Human energy requirements. Food and nutrition bulletin 2005; 63-66.

[11] Dhaka A. BANGLADESH DEMOGRAPHIC AND HEALTH SURVEY 2014 National Institute of Population Research and Training Ministry of Health and Family Welfare Dhaka, Bangladesh, www.mitra.bd.com. (2016).

[12] IPHN. State of Food security and nutrition in Bangladesh, www.sph.bracu.ac.bd. (2015).

[13] BBS. Preliminary Report on Household Income and Expenditure Survey 2016 BANGLADESH BUREAU OF STATISTICS (BBS) STATISTICS AND INFORMATICS DIVISION (SID) MINISTRY OF PLANNING GOVERNMENT OF THE PEOPLE'S REPUBLIC OF BANGLADESH. 2016.

[14] BBS. National hygiene survey 2018. 2018.

[15] Kumar Das S, Golam Faruque AS, Ahmed S. Nutritional and Micronutrient Status of Elderly People Living in a Rural Community of Bangladesh. Journal of Gerontology \& Geriatric Research; 01. Epub ahead of print 2015. DOI: 10.4172/21677182.1000107.

[16] Amugsi DA, Dimbuene ZT, Kyobutungi C. Correlates of the double burden of malnutrition among women: An analysis of cross-sectional survey data from sub-Saharan Africa. BMJ Open; 9. Epub ahead of print July 1, 2019. DOI: 10.1136/bmjopen2019-029545.

[17] Das S, Gulshan J. Different forms of malnutrition among under five children in Bangladesh: A cross sectional study on prevalence and determinants. BMC Nutrition; 3. Epub ahead of print December 22, 2017. DOI: 10.1186/s40795-016-0122-2.

[18] Sartorius B, Veerman LJ, Manyema M, et al. Determinants of obesity and associated population attributability, South Africa: Empirical evidence from a national panel survey, 2008-2012. PLOS ONE; 10. Epub ahead of print June 10, 2015. DOI:

10.1371/journal.pone.0130218. 